\title{
Hydraulic analysis of EU-DEMO divertor plasma facing components cooling circuit under nominal operating scenarios
}

\author{
P. A. Di Maio ${ }^{\text {a }}$ S. Garitta ${ }^{\mathrm{a}}$, J. H. You ${ }^{\mathrm{b}}$, G. Mazzone ${ }^{\mathrm{c}}$, E. Vallone ${ }^{\mathrm{a}^{*}}$ \\ ${ }^{a}$ University of Palermo, Viale delle Scienze, Edificio 6, 90128 Palermo, Italy \\ ${ }^{b}$ Max Planck Institute of Plasma Physics (E2M), Boltzmann Str.2, 85748 Garching, Germany \\ ${ }^{c}$ Department of Fusion and Technology for Nuclear Safety and Security, ENEA C.R. Frascati, via E. Fermi 45, 00044 \\ Frascati (Roma), Italy
}

Within the framework of the Work Package DIV 1 - "Divertor Cassette Design and Integration" of the EUROfusion action, a research campaign has been jointly carried out by University of Palermo and ENEA to investigate the steady state thermal-hydraulic behaviour of the DEMO divertor cassette cooling circuit, focussing the attention on its Plasma Facing Components (PFCs). The research campaign has been carried out following a theoretical-computational approach based on the Finite Volume Method and adopting the commercial Computational Fluid-Dynamic code ANSYS-CFX.

A realistic model of the PFCs cooling circuit has been analysed, specifically embedding each Plasma Facing Unit (PFU) cooling channel with the foreseen swirl tape turbulence promoter, hence resulting in a finite volume model much more detailed than those assessed in previous analyses. Its thermal-hydraulic performances have been numerically evaluated under nominal steady state conditions, also comparing the obtained results with the corresponding outcomes of analogous analyses carried out for a simplified PFCs configuration, without swirl tapes. Moreover, the main thermal-hydraulic parameters have been evaluated in order to check whether the considered PFCs cooling circuit might fulfil the total pressure drop requirement $(\Delta \mathrm{p}<1.4 \mathrm{MPa})$, providing a uniform cooling of the Vertical Target PFU channels with a viable CHF margin ( $>1.4)$.

The PFCs cooling circuit thermal-hydraulic behaviour has been additionally assessed at alternative operative conditions, issued to check the viability of a coolant velocity reduction, in order to minimize corrosion and vibrations inside the PFU channels.

Models, loads and boundary conditions assumed for the analyses are herewith reported and critically discussed, together with the main results obtained.

Keywords: DEMO, divertor, plasma facing components, CFD analysis, thermofluid-dynamics.

\section{Introduction}

Within the framework of the activities foreseen by the WP-DIV 1 - "Divertor Cassette Design and Integration" [1] of the EUROfusion action, a research campaign has been jointly carried out by University of Palermo and ENEA to investigate the steady state thermal-hydraulic behaviour of the DEMO divertor Plasma Facing Components (PFCs) cooling circuit $[2,3]$.

In particular, a realistic model of the PFCs cooling circuit has been developed. Its thermal-hydraulic performances have been numerically evaluated under steady state reference and alternative conditions, the latter being issued to check the viability of a coolant velocity reduction, in order to minimize corrosion and vibrations inside the Plasma Facing Unit (PFU) channels. Moreover, the main thermal-hydraulic parameters have been evaluated in order to check whether the considered PFCs cooling circuit might fulfil the total pressure drop requirement $(\Delta \mathrm{p}<1.4 \mathrm{MPa})$, providing a uniform cooling of the vertical target PFU channels with a viable CHF margin (> 1.4).

The research campaign has been performed following a computational approach based on the finite volume method and adopting the ANSYS CFX v.16.2 Computational Fluid-Dynamic (CFD) code, also used to evaluate hydraulic resistances for system codes $[4,5,6]$.
Analysis models and assumptions are herein reported and critically discussed, together with the main results obtained.

\section{Outline of DEMO divertor cassette}

According to its 2016 design, DEMO divertor is articulated in 54 toroidal cassettes, each composed of a Cassette Body (CB) supporting two PFCs, namely an Inner Vertical Target (IVT) and an Outer Vertical Target (OVT) (Fig. 1), composed of actively cooled PFUs equipped with a Swirl Tape (ST) turbulence promoter.

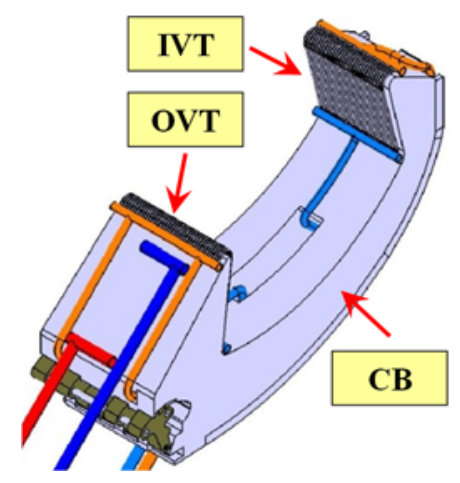

Fig. 1. DEMO divertor cassette 2016 design. 


\section{PFCs cooling circuit}

The analysed PFCs cooling circuit has originated from the optimisation of its layout, issued during the second half of 2016 [7] and thoroughly described in [2]. In particular, if compared with the original layout, manifolds diameter has been increased by a factor 1.4 and properlyshaped diffusers between VTs manifolds and their inlet headers have been introduced.

However, in order to save computational effort, the optimisation was carried out on a simplified model, mainly characterised by the absence of the swirl tape turbulence promoter inside the PFUs cooling channels, whose contribution to the total pressure drop was separately assessed by a proper correlation and added to the code-calculated one in the post-processing phase.

A more realistic assessment of the thermal-hydraulic performances of the optimised PFCs cooling circuit layout has been, hence, pursued, adopting a more onerous model embedded with swirl tape turbulence promoters. In particular, it is characterised by $31 \mathrm{PFU}$ channels in the IVT and 39 in the OVT and by two separate OVT outlet manifolds (Fig. 2). Further details on structural and functional materials may be found in $[1,2,3]$.

The considered PFCs cooling circuit relies on the use of subcooled pressurized water at the inlet pressure and temperature of $5 \mathrm{MPa}$ and $130^{\circ} \mathrm{C}$, respectively, flowing under quasi-isothermal conditions.

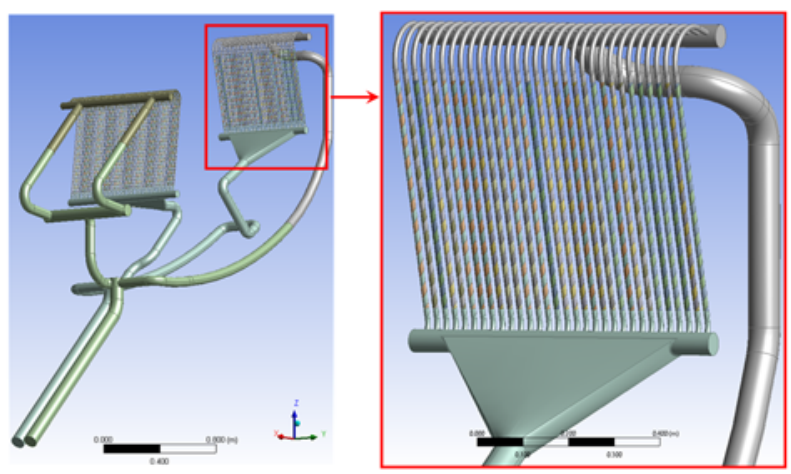

Fig. 2. PFCs cooling circuit analysed during 2017.

\section{PFCs cooling circuit CFD analysis}

Initially, the thermal-hydraulic behaviour of this PFCs cooling circuit layout option has been assessed assuming the reference coolant operative conditions agreed in October 2016 with EUROfusion teams.

Later on, the so-called "alternative" operating conditions have been considered. These conditions have been obtained from the former ones with the aim to reduce corrosion inside the PFU channels. As a consequence, also the inlet temperature has been decreased, so to allow the new CHF margin distribution to fulfil the prescribed requirement. Its value has been set to $90{ }^{\circ} \mathrm{C}$. The two considered coolant operative conditions are summarised in Table 1.
Table 1. Summary of coolant operative conditions.

\begin{tabular}{lcc}
\hline & $\begin{array}{c}\text { Reference } \\
\text { conditions }\end{array}$ & $\begin{array}{c}\text { Alternative } \\
\text { Conditions }\end{array}$ \\
\hline Inlet Pressure [MPa] & 5.0 & 5.0 \\
Inlet Temperature $\left[{ }^{\circ} \mathbf{C}\right]$ & 130 & 90 \\
$\Delta \mathbf{T}\left[{ }^{\circ} \mathbf{C}\right]$ & 6 & 9 \\
Removed Power $[\mathbf{M W}]$ & 136 & 136 \\
G per Cassette $[\mathrm{kg} / \mathrm{s}]$ & 98.63 & 67.56 \\
\hline
\end{tabular}

The thermal-hydraulic performances of the PFCs cooling circuit under coolant operative conditions of Table 1 have been assessed by running steady state, isothermal CFD analyses, assuming the coolant to flow at the average temperature between inlet and outlet sections of the cooling circuit.

Selected mesh parameters and main assumptions, models and Boundary Conditions (BCs) adopted are reported in Tables 2 and 3, respectively.

Table 2. Summary of selected mesh parameters.

\begin{tabular}{lc}
\hline Nodes & $6.305 \cdot 10^{+7}$ \\
Elements & $8.035 \cdot 10^{+7}$ \\
Inflation Layers Number & 12 \\
First Layer tThickness $[\boldsymbol{\mu m}]$ & 12 \\
Layers Growth Rate & 1.4 \\
Typical Element Size $[\mathbf{m}]$ & $2.18 \cdot 10^{-3}$ \\
Min/Avg/Max $\mathbf{y}^{+}$ & $2.972 / 112.3 / 496.3$ \\
\hline
\end{tabular}

Table 3. Summary of assumptions, models and BCs.

\begin{tabular}{lcc}
\hline & $\begin{array}{c}\text { Reference } \\
\text { conditions }\end{array}$ & $\begin{array}{c}\text { Alternative } \\
\text { Conditions }\end{array}$ \\
\hline Analysis Type & Steady state & Steady state \\
Material Library & IAPWS IF97 & IAPWS IF97 \\
Temperature & $133^{\circ} \mathrm{C}$ & $95^{\circ} \mathrm{C}$ \\
Turbulence Model & $\mathrm{k}-\varepsilon$ & $\mathrm{k}-\varepsilon$ \\
Boundary Layer & $\begin{array}{c}\text { Scalable wall } \\
\text { functions }\end{array}$ & $\begin{array}{c}\text { Scalable wall } \\
\text { functions }\end{array}$ \\
$\begin{array}{l}\text { Wodelling } \\
\begin{array}{l}\text { Inll Roughness } \\
\text { (Static Pressure) }\end{array}\end{array}$ & $2 \mu \mathrm{m}$ & $2 \mu \mathrm{m}$ \\
$\begin{array}{l}\text { Outlet BC } \\
\text { (Mass Flow Rate) }\end{array}$ & $98.63 \mathrm{~kg} / \mathrm{s}$ & $67.56 \mathrm{~kg} / \mathrm{s}$ \\
\hline
\end{tabular}

\section{Results at reference operative conditions}

The coolant total pressure spatial distribution within the PFCs cooling circuit assessed at the reference operative conditions is reported in Fig. 3, while the corresponding total pressure drops across the main sections of the circuit are reported in Table 4.

The PFCs cooling circuit overall total pressure drop amounts to $\sim 1.1 \mathrm{MPa}$, resulting lower than the prescribed limit of 1.4 MPa. 


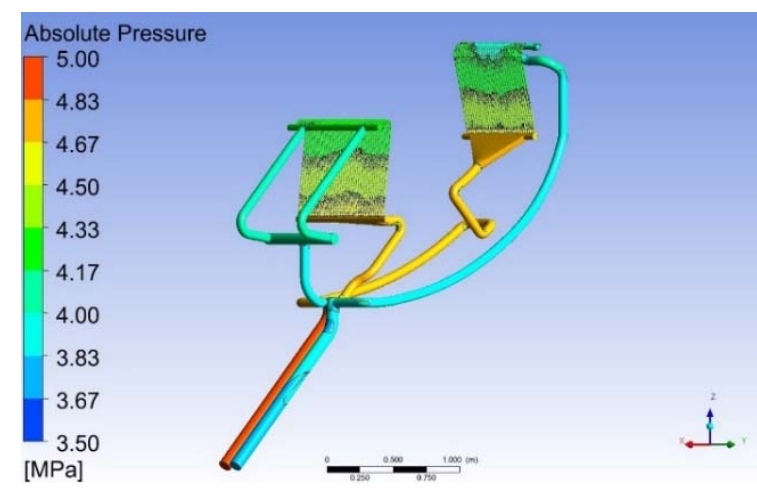

Fig. 3. Total pressure field (reference conditions).

Table 4. Total pressure drops (reference conditions).

\begin{tabular}{lc}
\hline Sections & $\Delta \mathrm{p}[\mathrm{MPa}]$ \\
\hline Inlet Common Manifold & 0.0131 \\
Inlet IVT Wye & 0.1239 \\
Inlet IVT Manifold & 0.0996 \\
IVT & 0.6855 \\
Outlet IVT Manifold & 0.0463 \\
Outlet IVT Wye & 0.0891 \\
IVT Circuit Total & $\mathbf{1 . 0 4 4 4}$ \\
Inlet OVT Wye & 0.1172 \\
Inlet OVT Manifold & 0.0623 \\
OVT & 0.6424 \\
Outlet OVT Manifolds I & 0.0091 \\
Outlet OVT Manifold II & 0.0870 \\
Outlet OVT Wye & 0.1266 \\
OVT Circuit Total & $\mathbf{1 . 0 4 4 4}$ \\
Outlet Common Manifold & 0.0315 \\
PFCs Circuit TOTAL & $\mathbf{1 . 0 8 9 0}$ \\
\hline
\end{tabular}

Moreover, attention has been paid also to the sectionaveraged values of coolant axial flow velocity $\left(\mathrm{V}_{\mathrm{ax}}\right)$ within the PFU channels of both OVT and IVT. In particular, the distributions of $\mathrm{V}_{\mathrm{ax}}$ among the VTs PFU channels are shown in Fig. 4 and their key-parameters have been reported in Table 5 .

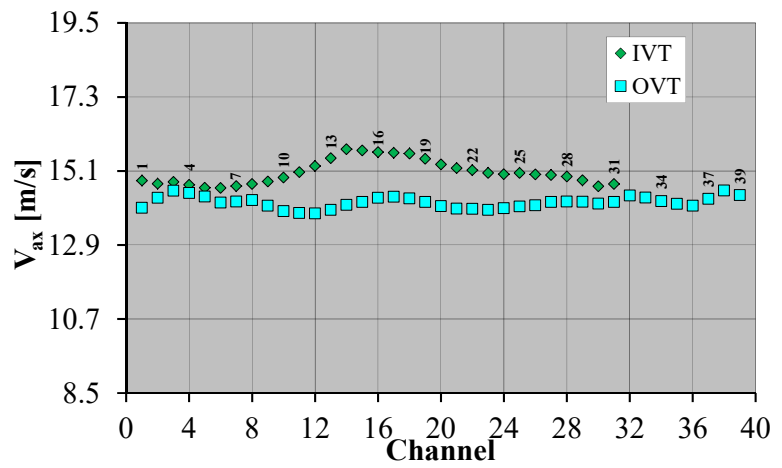

Fig. 4. $V_{\text {ax }}$ distribution among PFU channels (reference conditions).
Table 5. $V_{\text {ax }}$ distribution key-parameters (reference conditions).

\begin{tabular}{lcc}
\hline & OVT & IVT \\
\hline $\operatorname{Max}_{\text {ax }}[\mathbf{m} / \mathbf{s}]$ & 14.517 & 15.746 \\
$\operatorname{Min}_{\mathbf{a x}}[\mathbf{m} / \mathbf{s}]$ & 13.837 & 14.595 \\
EMax-Min $\left._{\text {Average }} \mathbf{V a x}_{\text {am }} / \mathbf{s}\right]$ & $4.68 \%$ & $7.31 \%$ \\
$\sigma[\mathbf{m} / \mathbf{s}]$ & 14.161 & 15.053 \\
\hline
\end{tabular}

From the analysis of the results obtained, it may be argued that within the PFU channels of each VT the distribution of $\mathrm{V}_{\mathrm{ax}}$ is quite uniform, since maximum deviations lower than $8 \%$ have been estimated between the maximum ( $M a x V_{a x}$ ) and minimum (Min $V_{a x}$ ) values.

The distributions of the margin against CHF onset within the VTs PFU channels have been assessed for the PFCs cooling circuit, mainly in order to check whether its prescribed minimum value of 1.4 is guaranteed by the cooling circuit layout. To this purpose, attention has been paid to the strike point sections of both OVT and IVT, where it has been supposed to be located the peak value of the incident heat flux arising from plasma (assumed equal to $20 \mathrm{MW} / \mathrm{m}^{2}$ according to [3]). In these sections, water coolant has been supposed to flow at the temperature of $133{ }^{\circ} \mathrm{C}$, with the local values of total pressure and axial flow velocity previously calculated for each VTs PFU channel. In these hypotheses, the CHF at the interface between the coolant and the channel walls has been calculated for each VTs PFU channel by means of the correlation given in [8]. CHF margin distributions have been reported in Fig. 5, while key-parameters have been summarized in Table 6 .

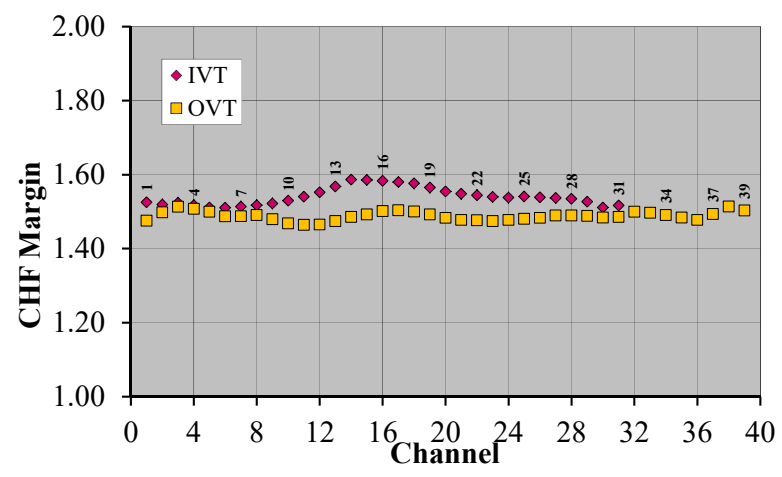

Fig. 5. CHF margin distribution among PFU channels (reference conditions).

Table 6. CHF margin distribution key-parameters (reference conditions).

\begin{tabular}{lcc}
\hline & OVT & IVT \\
\hline Max CHF Margin & 1.513 & 1.586 \\
Min CHF Margin & 1.464 & 1.510 \\
EMax-Min & $3.27 \%$ & $4.80 \%$ \\
Average CHF Margin & 1.488 & 1.540 \\
$\sigma$ & 0.012 & 0.024 \\
\hline
\end{tabular}


From the results obtained, it may be argued that the CHF margin distributions are acceptably uniform, since deviations between their maximum and minimum values are less than 5\%. Moreover, minimum values calculated for both VTs are higher than the prescribed value of 1.4.

\section{Results at alternative operative conditions}

The alternative conditions have been issued to check the viability of a coolant velocity reduction (to less than $12 \mathrm{~m} / \mathrm{s}$ [9]) in order to minimize corrosion. Moreover, the inlet temperature has been reduced to $90^{\circ} \mathrm{C}$, as it has been derived to be the minimum value allowing $\mathrm{CHF}$ margin distributions to overcome the limit of 1.4.

Total pressure spatial distribution and total pressure drops distribution are reported in Fig. 6 and Table 7, respectively. PFCs cooling circuit total pressure drop amounts to $\sim 0.5 \mathrm{MPa}$, about one half of the value calculated at reference conditions. $\mathrm{V}_{\mathrm{ax}}$ distributions among PFU channels have been reported in Fig. 7, summarising their main parameters in Table 8 .

Table 7. Total pressure drops (alternative conditions).

\begin{tabular}{lc}
\hline Sections & $\Delta \mathbf{p}[\mathrm{MPa}]$ \\
\hline Inlet Common Manifold & 0.0063 \\
Inlet IVT Wye & 0.0637 \\
Inlet IVT Manifold & 0.0409 \\
IVT & 0.3231 \\
Outlet IVT Manifold & 0.0234 \\
Outlet IVT Wye & 0.0406 \\
IVT Circuit Total & $\mathbf{0 . 4 9 1 7}$ \\
Inlet OVT Wye & 0.0538 \\
Inlet OVT Manifold & 0.0320 \\
OVT & 0.2983 \\
Outlet OVT Manifolds I & 0.0043 \\
Outlet OVT Manifold II & 0.0429 \\
Outlet OVT Wye & 0.0603 \\
OVT Circuit Total & $\mathbf{0 . 4 9 1 7}$ \\
Outlet Common Manifold & 0.0114 \\
TOTAL & $\mathbf{0 . 5 0 9 3}$ \\
\hline
\end{tabular}

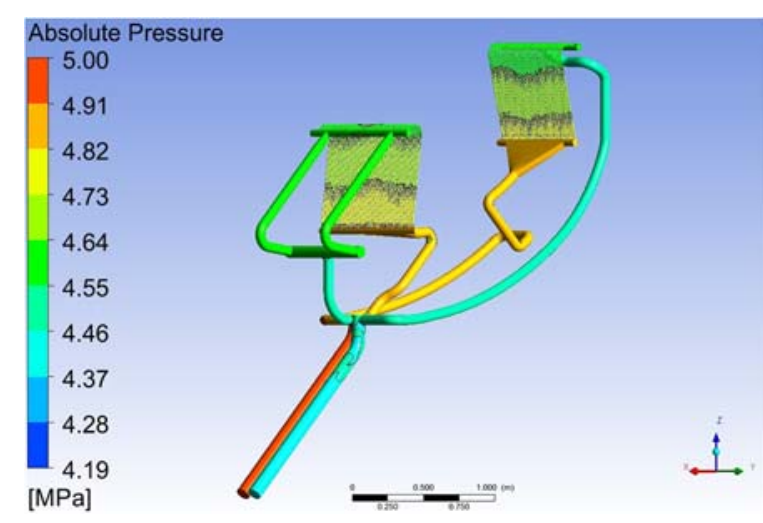

Fig. 6. Total pressure field (alternative conditions).

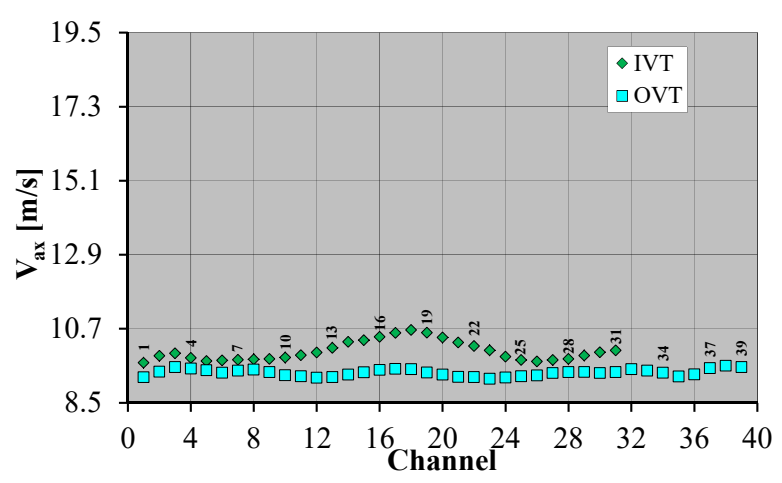

Fig. 7. $\mathrm{V}_{\mathrm{ax}}$ distribution among PFU channels (alternative conditions).

Table 8. $\mathrm{V}_{\text {ax }}$ distribution key-parameters (alternative conditions).

\begin{tabular}{lcc}
\hline & OVT & IVT \\
\hline $\operatorname{Max}_{\mathbf{a x}}[\mathbf{m} / \mathbf{s}]$ & 9.599 & 10.663 \\
$\operatorname{Min}_{\mathbf{a x}}[\mathbf{m} / \mathbf{s}]$ & 9.212 & 9.689 \\
EMax-Min $_{\text {Average } \mathbf{V}_{\text {ax }}[\mathbf{m} / \mathbf{s}]}$ & $4.04 \%$ & $9.14 \%$ \\
$\sigma[\mathbf{m} / \mathbf{s}]$ & 9.392 & 10.032 \\
\hline
\end{tabular}

From the results obtained, it may be argued that the distribution of section-averaged coolant axial flow velocity among VTs PFUs channels is quite uniform, since maximum deviations lower than $10 \%$ have been estimated between their maximum (Max $\mathrm{V}_{\mathrm{ax}}$ ) and minimum (Min $\mathrm{V}_{\mathrm{ax}}$ ) calculated values.

The CHF margin distributions among the VTs PFU cooling channels have been assessed according the procedure and assumptions of section 5. In particular, the obtained distributions have been reported in Fig. 8, while their key-parameters have been summarized in Table 9.

From the results obtained, it may be argued that the calculated distributions of CHF margin are acceptably uniform for both the VTs, since deviations between their pertaining maximum and minimum values amount to less than $6.5 \%$. Moreover, minimum values of CHF margin calculated for both VTs result higher than the prescribed limit of 1.4.

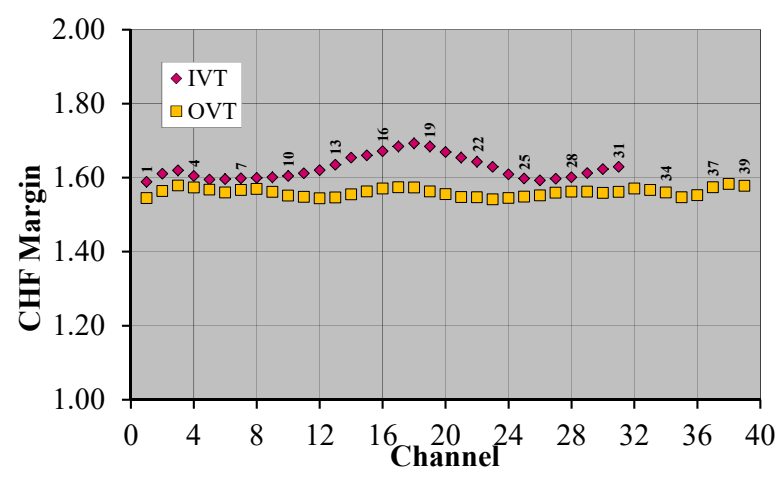

Fig. 8. CHF margin distribution among PFU channels (alternative conditions). 
Table 9. CHF margin distribution key-parameters (alternative conditions).

\begin{tabular}{lcc}
\hline & OVT & IVT \\
\hline Max CHF Margin & 1.583 & 1.693 \\
Min CHF Margin & 1.541 & 1.588 \\
EMax-Min & $2.64 \%$ & $6.16 \%$ \\
Average CHF Margin & 1.560 & 1.625 \\
$\sigma$ & 0.011 & 0.031 \\
\hline
\end{tabular}

\section{Conclusions}

Within the framework of the activities foreseen by the WP-DIV 1 "Divertor Cassette Design and Integration" of the EUROfusion action, a research campaign has been carried out at the University of Palermo, in cooperation with ENEA, to theoretically investigate the steady state thermal-hydraulic performances of the DEMO divertor PFCs optimised configuration. To this purpose, a theoretical-computational approach based on the finite volume method has been followed and the ANSYS CFX v.16.2 CFD code has been adopted.

The PFCs cooling circuit steady state thermalhydraulic performances have been numerically assessed in terms of coolant total pressure drop, flow velocity and CHF margin distributions, to check whether they comply with the corresponding reference limits.

The results of the CFD analysis have shown that the considered PFCs cooling circuit layout complies with the prescribed requirements. In particular, it has shown a total pressure drop of $1.0890 \mathrm{MPa}$, widely lower than the limit of $1.4 \mathrm{MPa}$, and a minimum margin against $\mathrm{CHF}$ occurrence of 1.464, higher than the limit of 1.4 . Moreover, this compliance has been also confirmed in case the cooling circuit would operate under alternative conditions, issued to check the viability of a coolant velocity reduction in order to minimize corrosion inside the PFU channels.

\section{Acknowledgments}

This work has been carried out within the framework of the EUROfusion Consortium and has received funding from the Euratom research and training programme 20142018 under grant agreement No 633053. The views and opinions expressed herein do not necessarily reflect those of the European Commission.

\section{References}

[1] J.H. You, et al., Progress in the initial design activities for the European DEMO divertor: Subproject "Cassette", Fusion Eng. Des. (2017), http://dx.doi.org/10.1016/j.fusengdes.2017.03.01.

[2] P.A. Di Maio, S. Garitta, J. H. You, G. Mazzone, E. Vallone, On the thermal-hydraulic optimization of DEMO divertor plasma facing components cooling circuit, Fusion Engineering and Design (2018), https://doi.org/10.1016/j.fusengdes.2018.05.032.
[3] P. A. Di Maio, S. Garitta, G. Mazzone, E. Vallone, DEMO Divertor - Thermo-hydraulic assessment report 2017, Final Report on Deliverable DIV-1-T004-D007, IDM Ref. N. EFDA_D_2MR3LR.

[4] P.A. Di Maio et al., Analysis of the steady state hydraulic behaviour of the ITER blanket cooling system, Fusion Eng. Des. $\quad 98-99 \quad$ (2015) 1470-1473, http://dx.doi.org/10.1016/j.fusengdes.2015.05.070.

[5] P.A. Di Maio et al., Numerical simulation of the transient thermal-hydraulic behaviour of the ITER blanket cooling system under the draining operational procedure, Fusion Eng. Des. 98-99 (2015) 1664-1667, http://dx.doi.org/10.1016/j.fusengdes.2015.01.024.

[6] P.A. Di Maio et al., On the hydraulic behaviour of ITER Shield Blocks \#14 and \#08. Computational analysis and comparison with experimental tests, Fusion Eng. Des. 109-111 (2016) 30-36.

[7] Divertor_CAD_Model_2017, EFDA_D_2MVPNG v1.0.

[8] A.R. Raffray et al., Critical heat flux analysis and R\&D for the design of the ITER divertor, Fusion Eng. Des. 45 (1999) 377-407.

[9] EUROfusion, personal communications. 